\title{
Simple system for mapping magnetic field errors in tori
}

\author{
P. M. Bellan \\ California Institute of Technology, Pasadena, California 91125
}

(Received 31 July 1986; accepted for publication 22 September 1986)

A simple inexpensive clectron beam/detector system is described. This system is capable of making vector maps of magnetic field errors over the minor cross section of toroidal plasma devices.

Slight $(<1 \%)$ toroidal magnetic field errors can cause loss of fast electrons or even the entire plasma hitting the wall in toroidal plasma devices. ${ }^{1,2}$ This note describes a simple, inexpensive yet precise electron beam/detector system for making vector maps of ficld errors.

As shown in Fig. 1, an electron gun and detector are mounted on a common support structure which can be translated both horizontally and vertically across the minor cross section of a torus. The translation is accomplished by lead screws driven by computer-controlled stepper motors, one for each axis of motion.

The electron gun cathode [sce Fig. 2(a)] consists of a 10-mil W wire bent in a hairpin shape and mounted in a double-bore alumina tube which in turn is mounted in a rectangular Macor block. The W wire is etched down ${ }^{3}$ to a $3-5$ mil diameter in the bend region so as to provide a highly localized emission source. Bolted to the sides of the Macor block are two molybdenum plates each with a $0.050 \mathrm{in}$. hole through which electrons can escape. By appropriate biasing of these plates electron beams either parailel or antiparallel to the toroidal field can be emitted (co- or counter-injection). Typical operating parameters are filament current 3 $\mathrm{A}$, bias -30 to $-300 \mathrm{~V}$, and emission $1-1000 \mu \mathrm{A}$ (after a 20 min burn-in period). The acceleration voltage and toroidal magnetic field have to be adjusted so that the displacement due to magnetic curvature drift centers the beam on the detector if there are no errors, $i$.e., the voltage and toroidal field must satisfy the relation

$$
h=17 \mathrm{~V}^{1 / 2} / B \text {, }
$$

where $h$ is the vertical distance in centimeters from the electron gun to the center of the detector array, $V$ is the beam

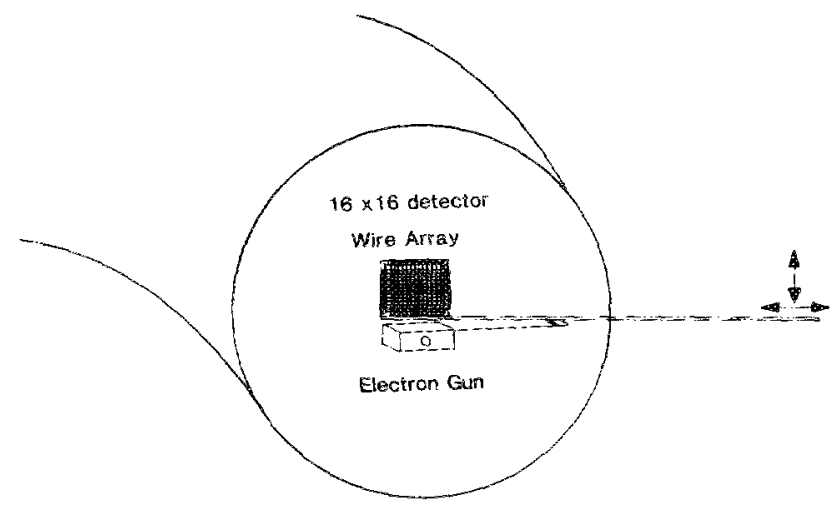

Fig. 1. Sketch of system. voltage, and $B$ is the magnetic field in gauss.

Because an electron beam is unaffected by pressures of a few times $10^{-5}$ Torr, it was possible to construct the detector ${ }^{4}$ from readily available commercial components. The detector consists of two orthogonal wire arrays as shown in Fig. 2(b). Each array consists of 16 segments of drill rod soft soldered onto the central 16 contacts of Ansley ribbon cable connectors ${ }^{5}$ which in turn are epoxied onto a Vector board frame. The Ansley connectors provide a precise $0.050 \mathrm{in}$. array spacing and the Vector board frame provides orthogonality.

A computer-controlled 32-channel analog multiplexer is used to measure the voltage developed across 32 resistors, one for each array wire [at low $(\sim 1 \mu \mathrm{A})$ emissions resistors of $15 \mathrm{MS}$ were used; at high emissions $(\sim 1000 \mu \mathrm{A})$ resistors of $47 \mathrm{k} \Omega$ were used]. To preserve high-frequency response the multiplexer is located only $\sim 1 \mathrm{~m}$ from the detec-

(a)

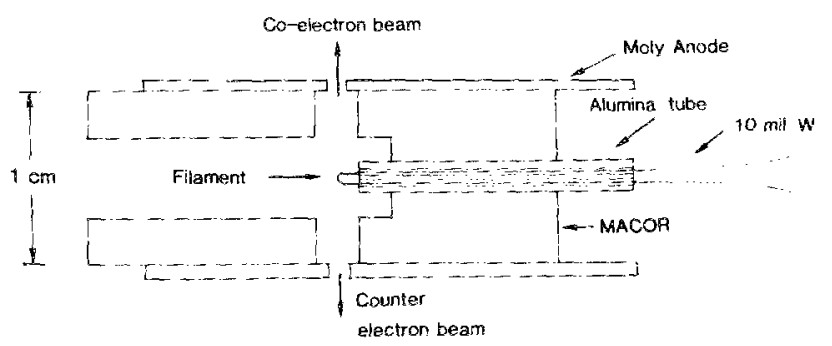

(b)

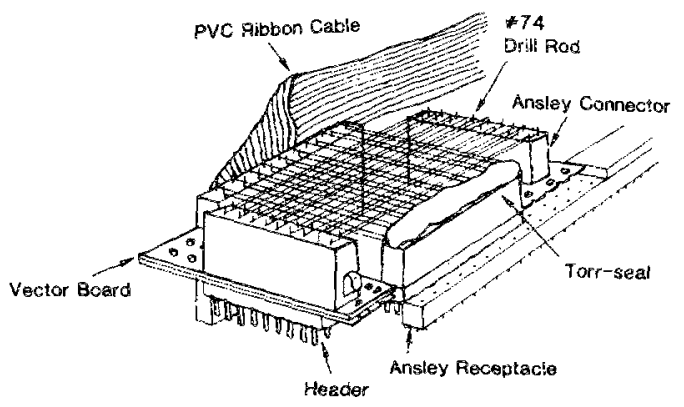

FiG. 2. (a) Electron gun cross section; (b) detector array (for clarity, only one of ribbon cables is shown). 


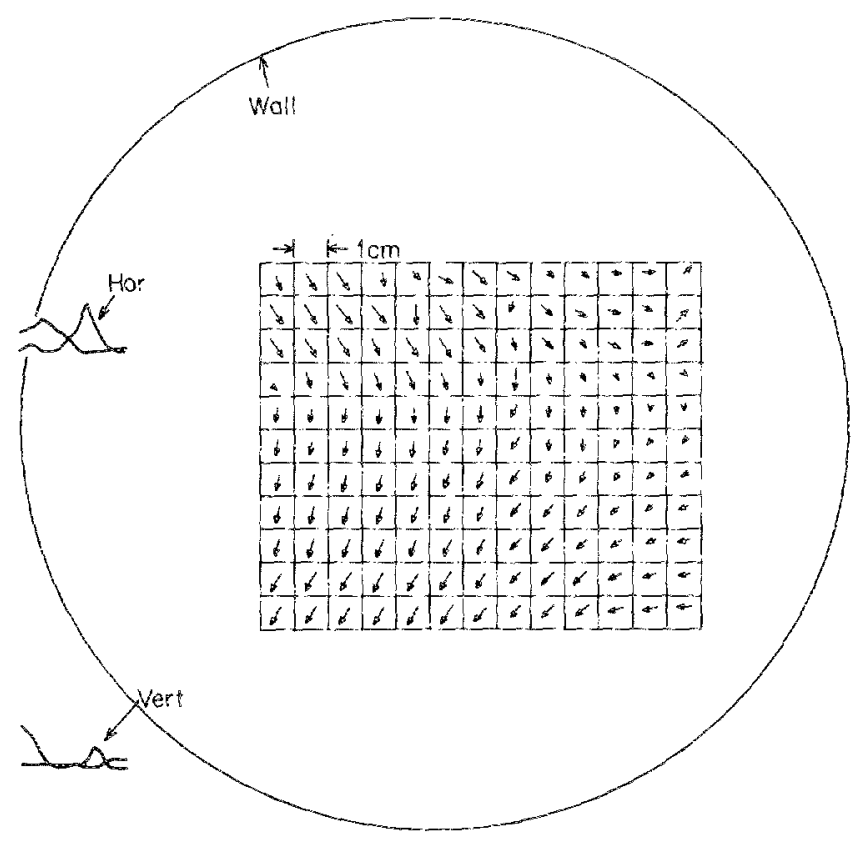

FrG. 3. Typical data, toroidal held $=76 \mathrm{G}$, beam voltage $=-30 \mathrm{~V}$, emis. sion $3 \mu \mathrm{A}$, Encore major radius $=0.38 \mathrm{~m}$, each square in plot is $1 \times 1 \mathrm{~cm}$.

tor array and a line driver is used to send the multiplexer along 50- $\Omega$ transmission line to a $12-$ bit $C A M A C A / D$ converter.

The computer makes a measurement first for a costreaming bean (one plate as anode, the other at cutofi), and then for a counter-streaming beam (reversed plates). If no field errors exist, then for both co-and counter-streaming electrons the vertical detector wires will show a signal peaked at the displacement $h$, while the horizontal detectors will show zero displacement.

If there is a vertical feld error, then the co-streaming electrons will have an additional upward displacement rela- tive to $h$, while the counter-streaming electrons will have an additional downward displacement. The difference between the displacements of the co- and counter-streaming drifts gives the vertical field error (times a factor of 2 ). Similarly, a horizontal error will cause the co-streaming beam to spiral inward, whereas the counter-streaming beam will spiral outward, so that the difference between the horizontal displacements gives the horizontal field error (again times a factor of 2 ).

The computer measures (for vertical and horizontal arrays separately) first the location of the co- and counterstreaming peaks, then calculates the difference between the two, and then plots these differences as a small vector on a graphics terminal. The vector is located at a spot corresponding to the beam/probe position. By moving the beam/ probe in faster fashion across the torus minor cross section and plotting the resultant vectors, a quantitative map of the error freld is produced.

Typical data are shown in Fig. 3. Here, the error fieid pattern turned out to be caused by a combination of the vertical component of the earth's magnetic field, and a permanent magnetic dipole located at $R=0, z=0$ (where there is a gap in the iron core of the Caltech Encore tokamak on which the measurement was made). The inset in Fig. 3 shows raw horizontal and vertical signals for co- and counter-streaming electrons for a probe positon corresponding to the lower left-hand corner of the field map.

The author would like to thank Frank Cosso for consiructing the multiplexer and anode switching system. This work was supported by DOE Grant DE-FG03-86ER 53232.

'T. Jernigan, R. Prater, and D. M. Meade, Phys. Fluids 14, 235 (1971). ${ }^{2}$ M. Okbayashi, V. Voitsenya, B. Ripin, J. Schmidt, and S. Yoshikawa, Phys. Fluids 16,1337 (1973).

${ }^{3}$ R. W. Motley, J. Appl. Phys. 43, 3711 (1972).

4T. Uckan, Rev. Sci. Instrum. 56, 90 (1985).

5Thomas and Betts Ansley ribbon cable connector, \#609-2001M. 